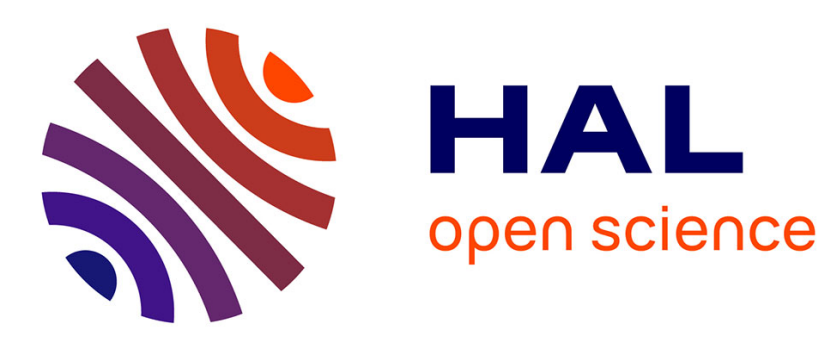

\title{
Kinking conditions for running cracks
}

N. Andrianopoulos, S. Kourkoulis

\section{To cite this version:}

N. Andrianopoulos, S. Kourkoulis. Kinking conditions for running cracks. Journal de Physique IV Proceedings, 1994, 04 (C8), pp.C8-777-C8-782. 10.1051/jp4:19948120 . jpa-00253363

\section{HAL Id: jpa-00253363 https://hal.science/jpa-00253363}

Submitted on 1 Jan 1994

HAL is a multi-disciplinary open access archive for the deposit and dissemination of scientific research documents, whether they are published or not. The documents may come from teaching and research institutions in France or abroad, or from public or private research centers.
L'archive ouverte pluridisciplinaire HAL, est destinée au dépôt et à la diffusion de documents scientifiques de niveau recherche, publiés ou non, émanant des établissements d'enseignement et de recherche français ou étrangers, des laboratoires publics ou privés. 
JOURNAL DE PHYSIQUE IV

Colloque C8, supplément au Journal de Physique III, Volume 4, septembre 1994

C8-777

\title{
Kinking conditions for running cracks
}

\author{
N.P. Andrianopoulos and S.K. Kourkoulis \\ National Technical University of Athens, Department of Engineering Science, Section of Mechanics, \\ 5 Heroes of Polytechnion Avenue, 157-73 Zografou, Athens, Greece
}

\begin{abstract}
Résumé: Le problème du changement abrupt de direction $d^{\prime}$ une fissure se propageant à grande vitesse est étudié dans le présent travail. L' étude est basée sur une aporoche unifiée des ohénomènes $d^{\prime}$ instabilité directionelle qui accompagne une fissure qui se propage. Selon cette approche, la pointe de fissure en propagation est simulée dar une configuration embranchée consistant en deux micro fissures de longueurs et $d^{\prime}$ orientations arbitraires. Ce $q u^{\prime}$ on voit en rêalité, $c^{\prime}$ est que les résultats macroscopiques dépendent de ces deux, a priori inconnues, longueurs et orientations des micro fissures, ainsi que du niveau des contraintes autour de la fissure et de sa vitesse.
\end{abstract}

\begin{abstract}
The problem of the abrupt change of the direction of a crack, propagating with high velocity, is studied in the present work. The study is based on a unified approach of the directional instability ohenomena, which accompany running cracks. According to this approach, the running tip is simulated by a prebranched configuration, consisted of two microcracks of arbitrary lengths and orientations. The final macroscopically observed result depends both on these, a oriori unknown, relative lengths and orientations of the two microcracks, and also on the stress level and crack velocity.
\end{abstract}

\section{INTRODUCTION}

The problem of abrupt change of the direction of fast running cracks (branching, curving and kinking), though one of the most popular problems in Linear Elastic Fracture Mechanics, still resists a satisfactory explanation and its complete understanding has not yet been achieved. The main reason for this $i 11$-success is that the problem lies on the borderline between micromechanics and continuum mechanics.

The criteria used for the study of these directional instability phenomena are, in general, based on the ideas introduced by Yoffe [1], according to which the distribution of a critical quantity around a single, mathematical crack will present, after a certain velocity limit, two off-axis extrema. This critical quantity may be a single stress [1], strain energy density [2,3] etc.

Unfortunately, these criteria fail to predict the deviation angle of the crack [4], though most of them have been succesfully applied in the static case, i.e. for the prediction of the initiation angle of a stationary crack [5]. The above observation and the experimentally verified fact, that from its early step of propagation the running tip is accompanied by a cloud of microcracks of random lengths and orientations [6,7], motivated Theocaris et aT. [8] to search the reasons of the above failure, not in the physical foundation of the criteria, but on their application mode.

Moving towards this direction, the "Twin-crack" model was introduced $[8,9]$. According to this approach, the moving crack is better simulated by a microscopically pre-branched configuration, consisted of two microcracks of different lengths and inclinations (Fig. 1). The further propagation of the micro-cracks, depends both on their relative lengths and orientations as well as the crack velocity and the stress level. 
The main advantage of this model, besides the improvement of the predictions of the criteria [8], is its remarkable flexibility to approach, in a unified manner, all the directional instability phenomena, i.e. branching, curving, kinking and, also, crack decceleration and arrest phenomena [9].

\section{THEORETICAL CONSIDERATIONS}

\subsection{The "Twin-crack" Model and the Kinking Problem}

According to the "Twin-crack" model the tip of a fast propagating crack is accompanied by a tuft of microcracks of random lengths and orientations having their origin in the activation of the imperfections in the structure of the material. At the final stages before macroscopic deviation, the tuft is converted into two crack fronts, accompanying the main crack tip. The orientation and dimensions of these fronts change continuously, as newly activated defects are absorbed by the moving fronts.

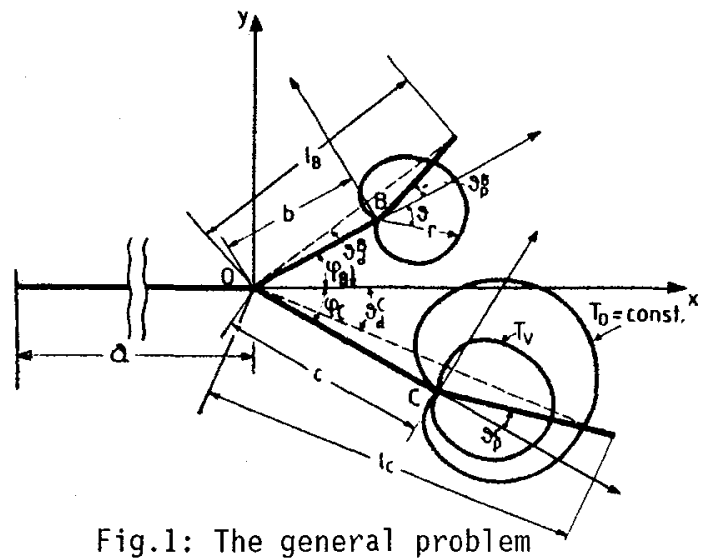

The abovedescribed complex configuration is simulated by a geometric shape, like the one shown in Fig.l, consisting of a main crack of length a and two microbranches of Tengths $b, c$ with $b, c<<a$ and inclinations $\varphi B$ and $\varphi c$ in relation to the main axis direction. It is further assumed that the two microbranches are moving with the same velocity, $u$, equal to the velocity of the mother crack. The future path of the two microbranches is defined by the instantaneous value of the angle between them, their lengths, the externally induced stress level and the achieved at that moment crack velocity. The further propagation angle of each branch, $\theta_{p}^{B} \cdot$, can be predicted by suitably applying any failure criterion around each one of the microbranches. Obviously, the macroscopically measured deviation angle, $\theta^{B C}$ is equal to:

$$
\theta^{B, C} \cong \varphi B, C+\theta^{B, C}
$$

provided that the restriction $1 B / b, 1 c / c<1$ holds $\left(F i g_{B}{ }_{C}\right)$. Depending on the specific relation between the angles $Q B, C$ and $\theta_{p}^{B}, C$, the angles $\theta_{d}^{B, C}$ are either positive or negative, leading the respective microbranch either to independent development or to absorption either by the main crack or by the other microbranch.

Summarizing, it can be stated that, for macroscopic crack path deviation to occur, the characteristic quantity of the failure criterion should exceed a threshold. However, this condition is necessary but not sufficient. At the same moment, the pair of microbranches should have the appropriate orientations and lengths, so that, at

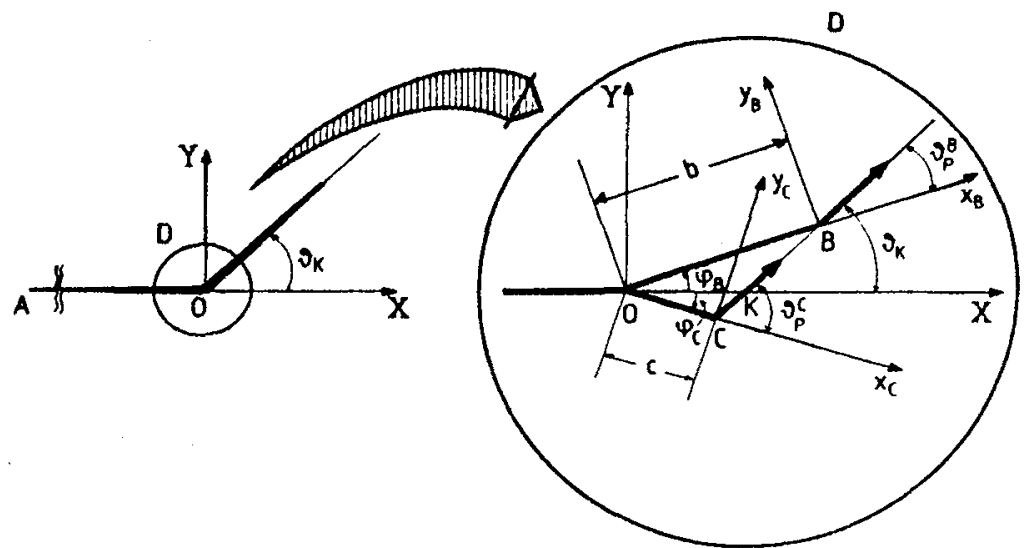

Fig. 2: The kinking configuration according to the "twin-crack" model 
least one of them can propagate independently. Hence, these phenomena are governed not by a single quantity, but rather by a six dimensional critical surface of the form:

$$
g\left(\sigma_{\infty}, u, b, c, \varphi B, \varphi c\right)=c_{0}
$$

where $0_{\infty}$ is the applied stress at infinity and co a material constant.

Concerning, thus, the specific problem of crack kinking, i.e. the abrupt change of the direction of the crack, it is obvious that, according to the "Twin-crack" model, it appears only in cases where one of the microbranches survives, while its counterpart coalesces with it or cannot initiate at a11. The macroscopically observable pattern, will then be that of a single crack with a kink in its direction and with slightly irregular lips in the area of direction change (Fig.2).

For the present study the T-criterion of failure is applied according to the "Twin-crack" mode1, since its predictions approach better experimental evidence [4].

\subsection{The Dynamic Version of the T-criterion}

The T-criterion of failure, initially proposed to study the crack initiation problem [10], when applied in Dynamic Fracture Mechanics is expressed as follows:

i) Each one of the two microbranches accompanying the main tip will propagate towards the direction $\theta_{p}^{\mathrm{B}, \mathrm{C}}$, at which the dilatational strain energy density $T \mathrm{~V}, \mathrm{ca} / \mathrm{cu}-$ lated along the Mises elastic-olastic boundary, $T_{0}=$ const., reaches a maximum value $T v_{\text {, }}$ ax, provided that this value exceeds a critical threshold TV.0, considered as material constant (Fig.1).

ii) Succesful macroscopic deviation is achieved for $\left(\varphi, \theta_{p}\right)$ combinations, allowing for the independent propagation of at least one of the microbranches.

For plane stress conditions the criterion is formulated as follows:

$$
\begin{gathered}
\left.T_{D}(r, \theta)\right|_{r=r(\theta)}=(1+v)\left(\sigma_{11}^{2}+\sigma_{22}^{2}-\sigma_{11} \sigma_{22}\right) / 3 E=T_{D, 0} \\
\left.\frac{\partial T_{v}(r(\theta), \theta)}{\partial \theta}\right|_{\theta=\theta}=0 \text { and }\left.\frac{\partial^{2} T_{v}(r(\theta), \theta)}{\partial \theta^{2}}\right|_{\theta=\theta}<0 \\
\left.T_{v}\left(r\left(\theta_{0}\right), \theta_{0}\right)\right|_{\theta=\theta}=(1-2 v)\left(\sigma_{11}+\sigma_{22}\right)^{2} / 6 E=T_{v, 0} \\
\theta_{d}^{B, C}=\varphi+\sin ^{-1}\left\{\sin \theta_{p}^{B, c}\left[\left(1-\left(m / T_{i}\right)^{2} \sin ^{2} \theta_{p}^{B, C}\right)^{1 / 2}-\frac{m}{T_{i}} \cos \theta_{p}^{B, C}\right]\right\} \cong\left(\varphi_{B, C}+\theta_{p}^{B, C}\right), m=b, c, 1_{i}=1_{B}, 1_{C}
\end{gathered}
$$

The application of the T-criterion necessitates knowledge of the dynamic stress field, i.e. the polar distribution and the stress intensity of it.

\subsection{The Dynamic Stress Field}

According to the solution given by Freund and Clifton [11], the stress components around cracks propagating with constant velocity are given by the following relations:

$$
\sigma_{i j}=\frac{K_{I}^{0}(t)}{\sqrt{2 \pi r}} \Gamma_{i j}^{I}\left(\theta, u, c_{1}, c_{2}\right)+\frac{K_{I I}^{0}(t)}{\sqrt{2 \pi r}} \Gamma_{i j}^{I I}\left(\theta, u, c_{1}, c_{2}\right)+0(1)
$$

with $(r, \theta)$ the polar coordinates system centered at the tip of the running crack, and $\Gamma_{i j}^{\lambda},(i, j=1,2$ and $\lambda=I, I I)$ analytic functions of angle $\theta$, the crack velocity $u$, and the respective dilatational and distortional stress waves velocities $\mathrm{c}_{1}$, c2, respectively.

In the same equation $K_{\lambda}^{\ell}(t)(\lambda=I, I I)$ are, respectively, the mode I and II, dynamic SIFs. Their value has been obtained by adopting the technique described by Kostrov [12], according to which the dynamic SIFs for propagating cracks are given as functions of their respective stationary value, $k_{\lambda}^{s}$ and of a correction factor $k \lambda$, which depends on the velocities of the crack and of the stress waves:

$$
K_{\lambda}^{0}(t)=K_{\lambda}^{S_{R}} k_{\lambda}^{\mu}(u), \quad k_{\lambda}^{\mu}=S(-1 / u)\left(1-\left(u / c_{r}\right)\right) /\left(1-\left(u / c_{\mu}\right)^{1 / 2}, \quad \lambda=I, I I, \quad \mu=1,2\right.
$$

In this equation $\mathrm{Cr}$ is the velocity of the Rayleigh stress waves and $S$ is a holomorphic function of crack velocity, calculated numerically.

Concerning the static SIFs, Theocaris [13] considered the three cracks of Fig.1 
as independent ones and obtained the following system of three complex singular integral equations:

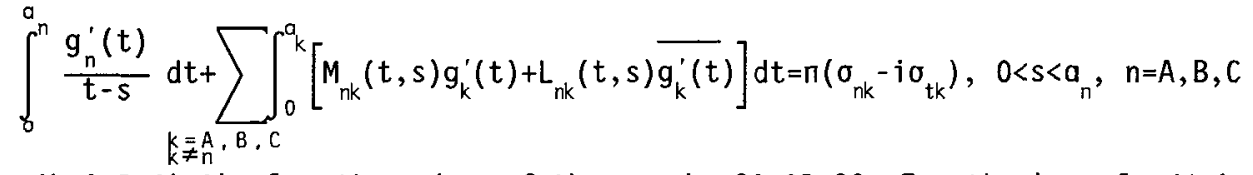

with ak $(k=A, B, C)$ the lengths $a, b, c$ of the cracks $O A, O B, O C$. For the kernels it holds:

$$
M_{n k}(t, s)=\bar{S}_{n k}(t, s)+S_{n k}(t, s) \exp \left[2 i\left(\theta_{k}-\theta_{n}\right)\right], L_{n k}(t, s)=S_{n k}(t, s)\left\{1-\frac{S_{n k}(t, s)}{\overline{S_{n k}}(t, s)} \exp \left[2 i\left(\theta_{k}-\theta_{n}\right)\right]\right\}
$$

with $S_{n k}=\left\{t-\operatorname{sexp}\left[i\left(\theta_{k}-\theta_{n}\right)\right]\right\}^{-1} / 2$, and $\sigma_{n k}-i \sigma t k=-\sigma\left[1+\exp \left(2 i \theta_{k}\right)\right] / 2$. The single-valuedness condition for the displacements around the composite crack gives the equation:

$$
\sum_{k=A, B, C}\left[\exp \left(i \theta_{k}\right) \int_{0}^{k} g_{k}^{\prime}(t) d t\right]=0
$$

The unknown functions $g_{k}^{\prime}(t)$ being proportional to the density of the edge-dislocations along the three branches are related to the complex SIFs through the relations:

$$
K_{k}=\sigma\left(2 n a_{k}\right)^{1 / 2} \lim _{s \rightarrow a_{k}}\left[\left(a_{k}-s\right)^{1 / 2} \bar{g}_{k}^{\prime}(s)\right], k=A, B, C
$$

However, $\bar{g}_{k}^{\prime}(s)$ can be written as :

$$
\bar{g}_{k}^{\prime}(s)=\left(a_{k}-s\right)^{1 / 2}\left[h_{k 1}(s)+i h_{k 2}(s)\right]
$$

where the real, $h_{k 1}(s)$ and the imaginary, $h_{k 2}(s)$, part of it have no singularities in $s=a k$. Hence, the complex SIFs are, finally, written as:

$$
k_{k}=\sigma\left(2 \pi a_{k}\right)^{1 / 2}\left[h_{k 1}\left(a_{k}\right)-i h_{k 2}\left(a_{k}\right)\right], k=A, B, C
$$

Solution of the so obtained complex singular integral system is achieved numerically by applying the Gauss-Legendre and Gauss-Lobatto integration rules. The first one was applied to the integral equation of the branch $O A$ with $N=30$ points of integration, while the second one to the integral equations of branches $O B$, $O C$ with the same number of integration points. Since the Gauss-Lobatto method has (N-1) collocation points, arising from the linear system of $6 \mathrm{~N}$ real unknowns, two real equations are missing. These are obtained from the condition of single-valuedness of displacements, Eq. (11).

The advantage of this method, beyond of the direct calculation of SIFs at the tips of the branches without any extrapolation, is that it is proved to be very accurate and stable, especially for extreme geometries (small angles $\varphi \mathrm{B}, \mathrm{C}$, and length ratios $b / c)$, which are of special importance for the kinking problem.

\section{APPLICATION OF THE METHOD FOR THE KINKING PROBLEM}

The stress field being given, the T-criterion can be applied according to the "Twin-crack" model. For that, conditions for observing the configuration of Fig. 2 are searched. Previous experience [14] indicates that this is possible only for strongly unequal angles $\varphi B, C$ and lengths $b, c$. Thus, the method was applied for $\varphi B=15^{\circ}, 30^{\circ}, 45^{\circ}$, $b / c=1 / 0.5,1 / 0.75,1 / 1.25$ and $\varphi c \in\left[2^{\circ}, 75^{\circ}\right]$. The initial conditions are summarized as:

i) Crack OA propagates with constant velocity $u$ in an infinite thin sheet, loaded at infinity, normally to the crack axis.

ii) Tio $O$ becomes suddenly stationary and two branches $O B$ and $O C$ of lengths $b$ and $c$ and inclinations $\varphi \mathrm{B}$ and $\varphi c$ appear, propagating with velocities equal to $U$.

The advantage of the above formulation is that it permits direct comparison between the two possible states of crack tips, i.e. to continue running as a single tip (tip A) or to deviate from its straight path being bifurcated or kinked (tip 0 ). For any given geometry $(b / c, \varphi B, \varphi c)$ and crack velocity $u$ at each one of the three tips of Fig. I there exists a direction towards which the local dilatational strain energy density, along the Mises elastic-plastic boundary, shows a maximum value $T v, \max (i=A, B, C)$. Thus the respective crack is expected, according to the T-criterion, to propagate towards this direction. Which one of the two patterns (single $A$ or double 0 ) will be realized, depends on energy consumption considerations, as it will be discussed next. For that, the maximum values of dilatational strain energy density at tips $B, C$ reduced to the 


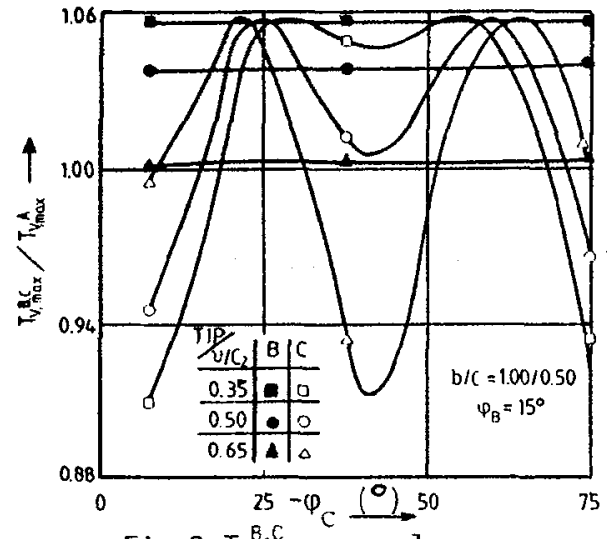

Fig. 3:TV,íax $v S$ angle $\varphi C$

respective value at tip $A$, are plotted, versus angle $\varphi c$, in $\mathrm{Fig.3}$, for $b / c=1 / 0.5, \varphi B=15^{\circ}$ and for three characteristic crack velocities, i.e. $\mathrm{v} / \mathrm{C}_{2}=0.35,0.50,0.65$.

It can be seen that, certain regions of $\varphi c$ angles exist, for which TV, max are greater than TA, max, implying that for such geometries and velocities the twin crack front 0 is more possible to appear and propagate than the single one, due to greater energy consumption.

Up to this point it seems that crack instability phenomena are completely stochastic not allowing for definite predictions concerning crack behaviour, because b, c, $\varphi$ s and $\varphi c$ cannot be a priori known. This contradicts to the common experience that, at least branching angles vary between narrow limits. It can be resolved by the present model which confines

optimum geometries for kinking to be observed. Such a confinement can be done through the hypothesis that a geometry is optimum for double patterns to appear when the sum of the dilatational strain energy consumption at the tips $B$ and $C$ becomes maximum. To locate these geometries we have plotted, in Fig.4, the quantity $\left(T v_{\text {, } \max }^{B} T T_{\text {, max }}{ }^{2}\right.$ for the same indicative geome- $u$ tries and velocities.It is clear from this $\mathrm{fi}-$ gure, that this quantity shows one or two maxima corresponding to the optimum combinations $(b, c, \phi B, \varphi c, u)$ for macroscopic crack path deviation and it has been proved that the first one corresponds to the usually observed symmetric or slightly asymmetric branching [8].

The question, now, is to which direction each branch will propagate or, in other words, which kind of crack instability will be realized. In the next Fig.5 the angular directions,

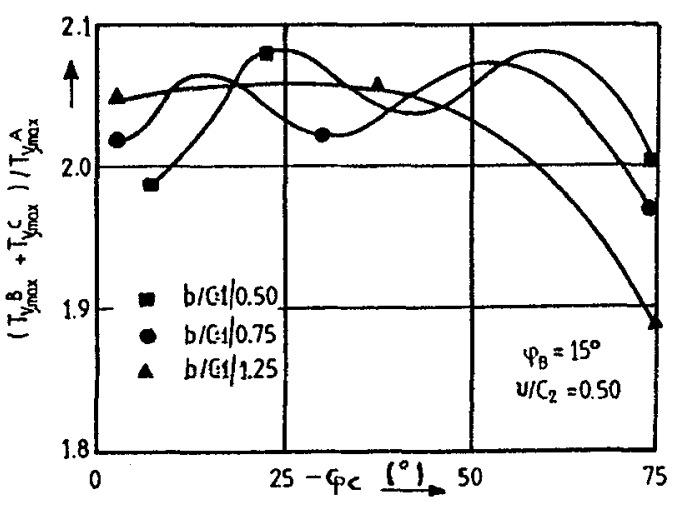

Fig. 4: The sum $T v^{B}, \max +T v^{C}$, ${ }^{C}$

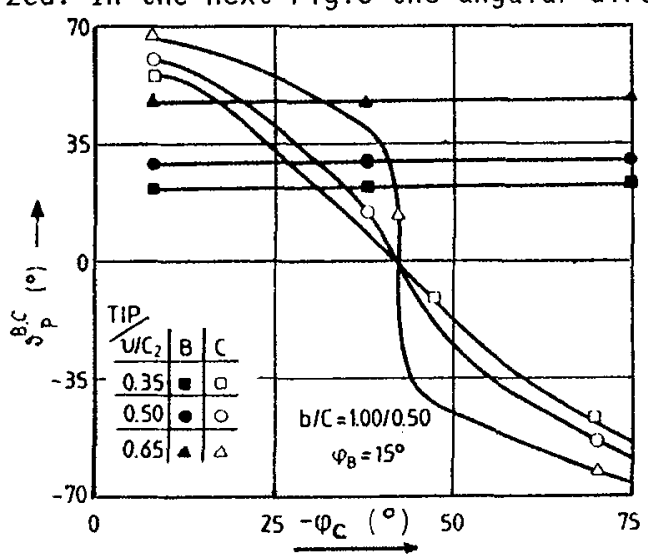

Fig.5: The propagation angles

$\theta_{p}^{B}$ and $\theta_{p}^{C}$, around the tips $B$, and $C$, where the maximum value of the dilatational strain energy density, $T V_{\text {, max }}^{B, C}$ is attained, are plotted, versus the initial angle $\phi c$, for the same as in Fig. 3 geometry and velocities. Both tips can propagate to either positive or negative directions with respect to their initial orientation. Kinking will be observed when both branches propagate along parallel directions i.e. When $\phi \mathrm{B}+\theta_{p} \cong \varphi c+\theta_{p}^{c}$, from $E q .(6)$, or when onty one branch propagates. This condition is sufficient in macroscopic terms because the lengths $b$ and $c$ are insignificant in comparison to the length a (see Fig.2).

Applying the above described procedure we locate combinations $(b, c, \varphi B, \phi c, u)$ leading to eventual macroscopic propagation of only one branch. For example, the combination $(b, c, \phi B$, $\varphi c, u)=\left(1,0.5,15^{\circ}, 7.5^{\circ}, 0.5 \mathrm{c} 2\right)$, resulting to angles $\theta_{p}^{B}=29.5^{\circ}$ and $\theta_{p}^{C}=60.5^{\circ}$, resembles, obviously, crack kinking, with kinking angle $\theta_{\mathrm{k}} \cong 44^{\circ}$. The results of such combinations are presented, synoptically, in next Fig.6, where kinking angle versus crack velocity has been plotted. It is observed that in spite of the uncertainty concerning the geometry of microbranches a rather narrow band of predictions is obtained. In addition limited experimental data from PMMA [15] are given, showing good agreement with the present predictions. 


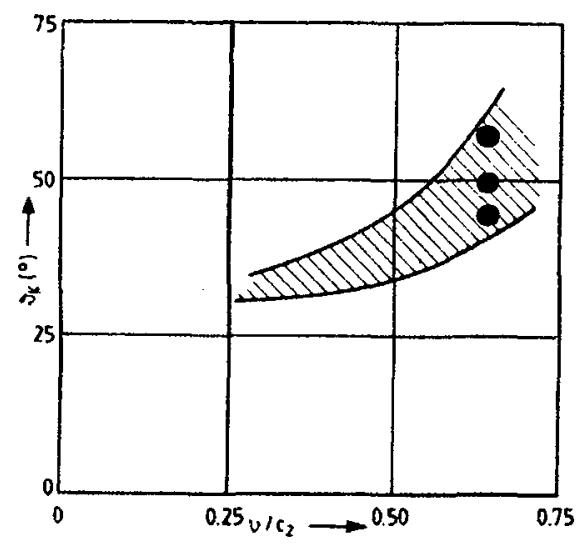

Fig.6: Kinking angle vs velocity

\section{DISCUSSION AND CONCLUSIONS}

The phenomenon of kinking has been studied in the present work by means of the T-criterion and the "Twin-crack" model, which permits a unified approach to all directional instability phenomena. This is achieved since, the a priori unknown, lengths and orientations of the microbranches act stochastically to the whole phenomenon, introducing an uncertainty factor. Thus, arbitrary assumptions, concerning their initial values, result in macroscopic geometries resembling all finally observed modes of dynamic crack path instability.

Concerning, the specific problem of crack kinking it is proved that it can be realized onty under extremely asymmetric geometries of the pre-branched configurations of the running crack tip. It implies that in a homogeneous isotropic material it is not very probable to expect kinking because of a relative lack of inhomogeneities. This observation agrees well with experimental evidence [16], that kinking appears more often in composites, while in homogeneous materials branching is the preferable deviation mode.

Also, it is indicated that the appearence of kinking is associated with decceleration of the running tip (or even momentary arrest), because real trajectory is longer than the macroscopically measured one, due to the longer sideway path followed (OKB $\angle O C B$ in Fig.2), a result clearly verified experimentally [16].

The final conclusion from the present study is that there does not exist a well defined mechanical quantity controlling the instability phenomena. Instead, a whole surface depending on both micro- and macroscopic factors, describes instabilities of running cracks. The microscopic level factors, being a priori unknown, are "suppressed" into two macroscopically interpretable quantities, i.e. the length ratio, $b / c$, of the two microbranches and the initial direction of them, QB.C. Although no predictions can be made on the exact values of them, the phenomenon is proved to be not completely random, since the demand of the model for maximum consumption of dilatational strain energy density defines optimum geometries prefered by the crack.

\section{REFERENCES}

[1] Yoffe E.H., Phil. Mag. 42 (1951) 739-750.

[2] Sih G.C., Mechanics of Fracture (G.C.Sih editor, Leyden, 1973) pp. XVII-XLVII.

[3] Theocaris P.S. and Georgiadis H.G., Int. J. Fracture 29 (1985) 181-190.

[4] Theocaris P.S., Andrianopoulos N.P. and Kourkoulis S.K., J. Pres. Ves. \& Piding 46 (1991) 149-166.

[5] Theocaris P.S., Andrianopoulos N.P. and Kourkoul is S.K., Exp. Mech. 27 (1987) $120-125$.

[6] Ravi-Chandar K. and Knauss W.G., Int. J. Fracture 26 (1984) 65-80.

[7] Theocaris P.S., Engng. Fract. Mech. 31 (1988) 255-270.

[8] Theocaris P.S., Andrianopoulos N.P. and Kourkoul is S.K., Engng. Fract. Mech. 34 (1989) 1097-1107.

[9] Andrianopoulos N.P. and Kourkoul is S.K., "A Unified Approach to the Crack Path Instability Phenomena", ICF8, Kiev June 1993.

[10] Theocaris P.S., Andrianopoulos N.P., Engng. Fract. Mech. 16 (1982) 425-432.

[11] Freund L.B. and CT ifton R.J., J. of Elasticity 4 (1974) 293-299.

[12] Kostrov B.V., Int. J. Fracture 11 (1975) 47-56.

[13] Theocaris P.S., J. App7. Mech. 44 (1977) 611-618.

[14] Theocaris P.S., Andrianopoulos N.P. and Kourkoul is S.K., Engng. Fract. Mech. 43 (1992) $137-146$.

[15] Theocaris P.S., Engng. Fract. Mech. 15 (1981) 283-290.

[16] Theocaris P.S. and Pazis D., Int. J. Solids Structures 19 (1983) 611-623. 